\title{
LA COMPETENCIA ESCRITA EN ASIGNATURAS \\ DEL CURRÍCULO DE LENGUA CASTELLANA. \\ COMPOSICIÓN DE TEXTOS ACADÉMICOS EN LA \\ FORMACIÓN DE MAESTROS.
}

\begin{abstract}
THE WRITTEN COMPETENCE IN SPANISH CURRICULUM SUBJECTS. ACADEMIC TEXTS COMPOSITIONS IN TEACHERS TRAINING.
\end{abstract}

La competencia escrita en asignaturas del currículo.

Juan David Zambrano-Valencia ${ }^{1}$ (D) ; Graciela Uribe-Álvarez ${ }^{2}$ (D); Zahyra Camargo-Martínez ${ }^{3}$ (D).

1.Universidad del Quindío. jdzambrano@uniquindio.edu.co.

2. Universidad del Quindío. guribe@uniquindio.edu.co.

3. Universidad del Quindío. zcamargo@uniquindio.edu.co.

*Correspondencia del Autor: Juan David Zambrano-Valencia, correo electrónico: jdzambrano@uniquindio.edu.co.

\section{RESUMEN}

Esta investigación se propuso optimizar los procesos de enseñanza y aprendizaje de habilidades escriturales en los estudiantes de dos asignaturas del currículo de español y literatura, mediante el diseño y aplicación de una propuesta de intervención para la producción de textos académicos (expositivo-explicativos y argumentativos), dirigida a alumnos de quinto y noveno semestre de la Licenciatura en Literatura y Lengua Castellana de la Universidad del Quindío, que buscó incidir en el desarrollo de la competencia escrita. La investigación es de corte cualitativo basado en el método de Elliott (2000) sobre Investigación-Acción-Participación (IAP). Como conclusión, se advierte que los profesores en formación logran planificar, producir, revisar, reescribir, editar y divulgar textos en sus asignaturas, a partir de la puesta en práctica de las estrategias propuestas, lo que redunda de manera positiva en su preparación como maestros y favorece el desarrollo de la competencia comunicativa.

Palabras clave: Competencia comunicativa; competencia escrita; estrategias; textos académicos.

Cómo citar:

Zambrano-Valencia, Juan David; Uribe-Álvarez, Graciela; Camargo-Martínez, Zahyra. (2020). La competencia escrita en asignaturas del currículo de lengua castellana. Composición de textos académicos en la formación de maestros. Revista de Investigaciones Universidad del Quindio, 32(2), 39-46. https://doi.org/10.33975/riuq.vol32n2.380 


\begin{abstract}
This research aimed to optimize the teaching and learning process of writing skills in students of two subjects in the curriculum of Spanish and literature, by designing and implementing an intervention proposal for the production of academic texts (Expositive-explanatory and argumentative) aimed at students in the fifth and ninth semesters of the bachelor's degree in Literature and Spanish Language from the University of Quindío which sought to influence the development of written competence. The research is qualitative based on the Elliott method (2000) on Research-Action-Participation (IAP). In conclusion, it is noted that teachers in training manage to planning, producing, reviewing, rewriting, editing and disseminate texts in their subjects, starting from the implementation of the proposed strategies, which has a positive effect on their preparation as teachers and benefits the development of communicative competence.
\end{abstract}

Keywords: Communicative competence; written competence; strategies; academic texts.

\section{INTRODUCCIÓN}

El desarrollo de la competencia escrita en el ámbito universitario se convierte en un propósito fundamental para la formación profesional $\mathrm{y}$ un reto investigativo que busca la cualificación de la escritura académica, de importancia capital en la escuela, la universidad y el entorno laboral, en general. Esta es una razón por la que la comunicación escrita cristaliza uno de los módulos de competencias genéricas de las pruebas Saber-Pro, obligatorio, como es bien sabido, para cualquier estudiante universitario, independiente de su disciplina. Así las cosas, tal competencia contribuye a la consolidación de la cultura académica, redunda en el avance de las ciencias y se traduce en un eslabón fundamental para la preparación de los maestros. De ahí que haya sido objeto de estudio en un abanico amplio de investigaciones del grupo Didáctica de la Lengua Materna y la Literatura - DiLeMa, en los últimos tres lustros (Camargo et al., 2006-2007; 2008-2009; 2011-2012; 2015-2016; Camargo et al., 2009- 2011; Zambrano et al., 2014-2015).

A la luz de tales pesquisas afloran hallazgos de los cuales inferimos que los estudiantes de la Licenciatura en Literatura y Lengua Castellana (LLLC) aún requieren de mediaciones que permitan, de forma sistemática, un tutelaje pertinente de los procesos de escritura académica (textos expositivo-explicativos y argumentativos) alimentado por estrategias inherentes al acceso al conocimiento, la planificación, la redacción, la revisión, la reescritura, la edición y la divulgación (Didactext, 2003,2015). Detalmanera, podríamos decir que la gestión de la competencia escrita se potencia y que los procesos de composición de textos académicos superan las dinámicas de aula, mediante propuestas de intervención que se traduzcan en una cualificación de la escritura.

El estudio de base se propuso, en materia general, optimizar los procesos de enseñanza y aprendizaje de la competencia escrita de los estudiantes de dos asignaturas del currículo de literatura y lengua castellana, quienes serán formadores del proceso escritural en el aula; y, en términos específicos, (i) diseñar una propuesta de intervención para la producción de textos expositivo-explicativos y argumentativos (contexto, actividades, estrategias, herramientas de ayuda y mediaciones que se llevan a cabo en cada una de las fases del proceso); (ii) aplicar la propuesta con estudiantes de los semestres $5^{\circ}$ y $9^{\circ}$ de la LLLC; (iii) generar un Recurso Educativo Digital Abierto -REDApara explicitar a futuros usuarios cómo llevar a cabo la propuesta de intervención en pro del desarrollo de las competencias en escritura de textos académicos; (iv) valorar los alcances del REDA, con el fin de determinar su posible uso en otros espacios académicos de la Licenciatura. 
En este artículo, por razones de espacio, solo enfatizamos en los objetivos específicos uno y dos, y en la contribución de las estrategias al desarrollo de la competencia escrita de los estudiantes.

Nos adherimos, por tanto, a la competencia comunicativa, que hace alusión a la habilidad que tiene el hablante para utilizar el sistema lingüístico de manera efectiva y apropiada, con inclusión no solo del conocimiento del sistema de reglas de una lengua, sino de cuándo, dónde, con quién y cómo utilizarlas. Desde la perspectiva de Hymes (1971), se tienen en cuenta factores pragmáticos, psicológicos y sociolingüísticos; mientras que Canale y Swain (1980) identifican tres componentes de esta competencia global: gramatical, sociolingüístico y estratégico. Así, ambas vertientes permiten entender la competencia comunicativa como un planteamiento provechoso para el aprendizaje y la enseñanza de la lengua materna. Gumperz y Hymes (1972), por su parte, en consonancia con Bachman (1995), sugieren que el conocimiento de la lengua requiere de dos grandes competencias: la organizacional y la pragmática, lo que nos lleva a pensar que la competencia comunicativa se erige, entonces, como un concepto abarcador que integra distintos repertorios que se proyectan, según circunstancias culturales, psicológicas y sociales.

En otros términos, el desarrollo de esta competencia exige un conocimiento, no sólo de tipo lingüístico, textual, sociocultural $\mathrm{y}$ pragmático, sino también, procedimental, lo que permite potenciar el uso del lenguaje. En este marco, asumimos el desarrollo de la competencia escrita, alrededor de estrategias involucradas con procesos cognitivos y metacognitivos, elementos motivacionales y afectivos, que es posible materializar en fases recursivas, según Didactext (2003, 2015), para el desarrollo de propuestas de intervención en las diversas áreas del currículo (Writing across the curriculum, Writing in the disciplines).

\section{METODOLOGÍA}

Desarrollamos una investigación con enfoque cualitativo basada en el método de Elliott (2000) sobre Investigación-Acción-Participación (IAP), quien lo define como el "estudio de una situación social para tratar de mejorar la calidad de la acción en la misma" (5). En general, en la IAP operan en paralelo la investigación pedagógica y la aplicación inmediata de los resultados, con el fin de intervenir y resolver problemas de aula reales y concretos. Con ello en mente, diseñamos una ruta metodológica estructurada en tres etapas y efectuamos la investigación con estudiantes del programa de LLLC de quinto semestre (29 alumnos) del espacio académico Didáctica de los textos argumentativos (EA1) y de noveno (22 alumnos) de la asignatura Didáctica de la comprensión y producción de textos funcionales (EA2), durante un semestre.

Etapa 1. Planificación. Relacionada con el diseño de la propuesta de intervención en lo que respecta a la organización del proceso de escritura académica de textos argumentativos (ensayos) y expositivo-explicativos (artículos divulgativos), tal como lo indicamos en el esquema:
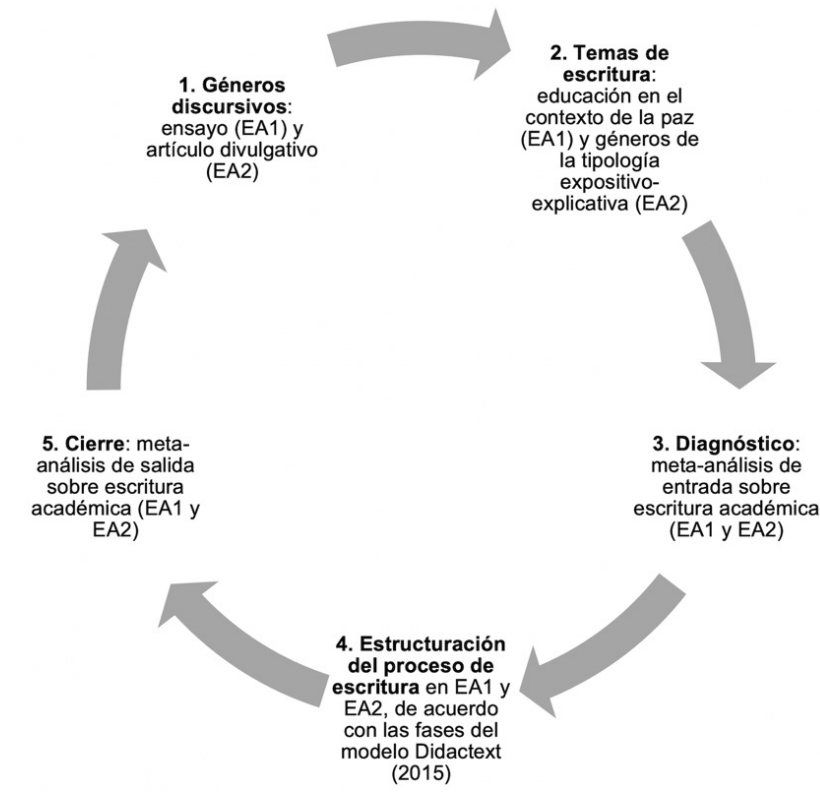

Esquema. Planificación del proceso de escritura 
Etapa 2. Experimental. Relacionada con el desarrollo de la propuesta de intervención. En ambas asignaturas se realizaron meta-análisis de entrada; en EA1, a partir de ensayos escritos en otros momentos de la vida académica de los estudiantes, y en EA2, mediante borradores de artículos divulgativos escritos in situ en 90 minutos. En EA1, revisamos 29 textos, mientras que en EA2, 16 (en este caso, los estudiantes podían escribir en parejas). Para ello acogimos un proceso de lectura cruzado-relacional que supone una revisión individual de cada producto, seguida de una lectura en paralelo de las partes constitutivas de los textos (Uribe et al., 2019). Gracias a los meta-análisis erigimos caminos de producción de ensayos y de artículos divulgativos que privilegian los diferentes tipos de revisión (individual, entre pares y colaborativa), en seis fases de escritura:

Fase 1. Acceso al conocimiento. Fundamentación teórica inherente a las categorías conceptuales de ambas asignaturas, a las características de las tipologías elegidas y de los géneros discursivos por escribir, así como a la exploración de la cuestión o búsqueda de información sobre las temáticas de los textos. En EA1, a la vez, exploramos una galería de tesis de los ensayos, para precisar cada una y ofrecer una ruta de búsqueda de textos relacionados con el tema específico.

Fase 2. Planificación. Solicitamos a los estudiantes un plan de escritura de los textos, en el que explicitaran el destinatario final de sus ensayos y artículos, organizaran la información obtenida en la fase anterior y determinaran la intencionalidad de los mismos. El producto final de esta fase, en los dos espacios académicos, fue un esquema que diera cuenta del plan de ideas o partitio de los ensayos y los artículos divulgativos, con base en la estructura de ambos géneros. En cuanto a los ensayos, además, los estudiantes debían expresar la tesis con claridad y adoptar la dispositio, en correspondencia con la tradición grecolatina (Aristóteles, 1986; Cicerón, 1997): exordio, narratio, confirmatio, peroratio.
Fase 3. Producción. Los estudiantes escribieron las primeras versiones de los ensayos y los artículos divulgativos, desde instrucciones de escritura concretas: Escriba un ensayo que no exceda las cinco páginas, en el que evidencie su postura sobre el papel de la educación en el contexto de la paz (EA1) y Escriban un artículo de divulgación sobre el género discursivo que han elegido para su intervención de aula (EA2).

Fase 4. Revisión y reescritura. En EA1, las primeras versiones de los ensayos fueron valoradas por el docente de la asignatura, de acuerdo con los criterios convenidos (Uribe et al., 2019). No obstante, a partir de allí, cada estudiante eligió un par evaluador (Carlino, 2009) para la revisión de la segunda versión, mientras que la tercera fue revisada de modo individual. En EA2, la primera versión de los artículos se valoró mediante comités editoriales que favorecieron la revisión en colaboración (Mercer y Littleton, 2007); la segunda corrió por cuenta de la lectura de pares externos no vinculados al espacio académico, mientras que la tercera la revisaron los docentes de las dos asignaturas. En EA1 y EA2, las versiones definitivas de los textos se usaron como base para el meta-análisis de salida.

Fase 5. Edición. En los dos casos orientamos la edición de los textos, de un lado, siguiendo el estilo APA, en lo que respecta a citación y construcción de referencias bibliográficas; y de otro, con base en criterios formales mínimos establecidos.

Fase 6. Divulgación. En EA1, los ensayos se leyeron en voz alta en diferentes encuentros en los que dimos lugar a discusiones de naturaleza argumentativa. En EA2, se presentaron oralmente con base en organizadores gráficos que los alumnos elaboraron para recoger las ideas-fuerza de los artículos.

En esta etapa de la ruta metodológica, además, los estudiantes firmaron consentimientos informados. 
Etapa 3. Diseño del REDA. Relacionada con la creación de un Recurso Educativo Digital Abierto-REDA-, denominado Expoargumentar, en el que se recogen las estrategias que diseñamos durante la investigación.

\section{RESULTADOS}

En este apartado presentamos los resultados con miras a determinar el aporte de la propuesta al desarrollo de la competencia escrita de los estudiantes, en su proceso de formación docente. A continuación, mostramos los resultados inherentes al diseño de una propuesta centrada en el modelo Didactext, en cada una de las asignaturas. En primer lugar, los resultados correspondientes a EA1:

En un primer momento, los ensayos revelaron problemas formales, estructurales y de contenido (títulos sin relación con el tema y la tesis; falta de apoyo en diversos argumentos para defender los puntos de vista; ensayos sin un esquema argumental; exceso de citas con poca exploración de otros argumentos o de contraargumentos; problemas morfosintácticos y notacionales; dificultades en la puntuación), lo que evidenciaba una posible falta de interés en las producciones escritas o poco acompañamiento de los profesores, tal vez, porque consideraban que ese conocimiento ya lo habían adquirido en otros niveles de su aprendizaje.

No obstante, en un segundo momento, después de la intervención, a pesar de que reconocemos que desconocen aspectos teóricos esenciales para la escritura de este tipo de textos, los resultados fueron muy diferentes: títulos contextualizados con el tema de la tesis; ensayos estructurados y defendidos con citas pertinentes; empleo apropiado de los argumentos, pero escaso uso de la contraargumentación; mejor manejo morfosintáctico, aunque con problemas de puntuación. Como queda expuesto, estas intervenciones didácticas favorecen, en este caso, la escritura de ensayos.
Ahora bien, en relación con EA2, los resultados fueron los siguientes:

Como en la anterior asignatura, los estudiantes, en la primera prueba, también presentaron dificultades formales, estructurales y de contenido (textos sin título o con un título que no reflejaba la macroestructura del texto; sin párrafos introductorios ni de síntesis; excesiva repetición de palabras; abandono de organizadores meta, intra e intertextuales, problemas morfosintácticos y notacionales; poco uso de citas o referencias bibliográficas). Estas problemáticas pueden tener como causa primaria que la mayoría de los docentes no tiene en cuenta, en sus procesos formativos, el desarrollo de estrategias didácticas que ayuden a sus estudiantes a hacer metacognición de sus aprendizajes, con el fin de llevar a la memoria a largo plazo las estructuras de los subtipos y de los diversos géneros discursivos, en este caso, de la tipología expositivo-explicativa.

Este panorama nos mostró la necesidad de llevar a cabo nuestra propuesta de intervención, con el fin de conseguir un cambio significativo en la elaboración de artículos divulgativos. El resultado de la segunda prueba fue muy satisfactorio: títulos que evidenciaban la macroestructura; uso de un subtipo definido; párrafos introductorios y de cierre; organizadores intra, meta e intertextuales; manejo adecuado de la gramática de la lengua, empleo de citas bien referenciadas y progresos en la puntuación; entre otras respuestas positivas. Debemos reconocer el acierto de ofrecerles el Modelo Didactext del que los estudiantes podían valerse para mirar la escritura como un proceso y no como un producto.

Por ejemplo, en la fase de edición solicitamos a los estudiantes, la revisión pormenorizada de sus textos con miras a una posible publicación; es decir, una escritura con un propósito y no un ejercicio de aula. Debemos aceptar, sin embargo, que a pesar de los avances, persistían algunas escrituras impropias; por eso, para cada problema elaboramos un reto que los obligara 
a hacer metacognición y a asumir sus falencias con ayuda también del REDA (Expoargumentar) diseñado para favorecer la escritura académica en soporte digital.

\section{DISCUSIÓN}

Podemos sostener, así, que la gestión de la competencia escrita de los estudiantes de EA1 y EA2 avanzó, porque se hicieron conscientes de algunos problemas de composición textual $\mathrm{y}$, gracias a procesos de metacognición, los enfrentaron estratégicamente, lo que, sin duda, dialoga con un conocimiento reflexivo y con un saber hacer deliberado que, en palabras de Gumpers y Hymes (1972: 7), se entiende como "aquello que un hablante necesita saber para comunicarse de manera eficaz en contextos socialmente significantes". Parece claro, entonces, que la mediación de los profesores y la voluntad de aprender de sus alumnos redundó de manera notoria en la preparación de estos docentes en formación y en el desarrollo de factores asociados con la competencia escrita: lo gramatical, inherente a las normas semánticas, sintácticas, morfológicas, fonológicas y fonéticas que rigen la producción de enunciados lingüísticos; lo sociolingüístico, que se refiere a reglas socioculturales de uso y adecuación de la forma al contexto; lo estratégico, que se ocupa de acciones planificadas relacionadas con la toma de decisiones, cuyo objeto se constituye por la efectividad de los procesos de comunicación (Canale y Swain, 1980). Por consiguiente, como Guerrero y Chois (2019), esperamos que las aportaciones de esta investigación:

contribuyan a avanzar en el campo disciplinario de los estudios sobre escritura en educación superior, pues aportan información sobre las prácticas de escritura en un espacio de formación y un campo disciplinario particular [...]. Son bienvenidos estudios que exploren y acompañen los esfuerzos de los programas académicos por transformar las prácticas docentes en beneficio de la formación escritural de los estudiantes (142).
También es posible decir que un número destacado de problemas de composición textual son de carácter teórico y que la relación entre epistemología y praxis facilita el aprendizaje de la escritura. Pensamos, entonces, que la propuesta didáctica fue oportuna. Además, discurrir sobre la función epistémica de la escritura conllevó a la toma de posición en calidad de integrantes de una comunidad académica que lee y escribe tipos de textos concretos, lo que, al tiempo, nos permitió efectuar prácticas pedagógicas con idoneidad. A propósito, Narváez (2017) ratifica la envergadura de investigaciones de esta índole:

a) no es una práctica común en el área hacer revisiones sistemáticas de literatura extranjera o metanálisis de los trabajos empíricos existentes, o b) no existen suficientes trabajos concentrados en una sola línea, o bases de datos organizando dichos trabajos que permitan o ameriten la creación de metanálisis. Por lo tanto, la producción de artículos de revisión (de literatura o metanálisis de trabajos empíricos) es un camino de producción de conocimiento en el campo que se requiere para sistematizar modelos teóricos y metodológicos, así como definiragendas de investigación especializadas (84).

\section{CONCLUSIONES}

La enseñanza de la escritura académica resulta tan decisiva en la universidad como el proceso mismo de composición de los aprendices; de allí que la formación consciente del profesorado en tal empresa no puede desligarse de la planificación intencionada ni del estudio de los modos específicos de escritura de cada disciplina $\mathrm{y}$, menos aún, de las creencias asociadas a las tradiciones epistemológicas y a las formas prototípicas usadas para la comunicación en cada ámbito social (géneros del discurso). Aunque parezca un lugar común del lenguaje, argüimos, por una parte, que el profesor interviene en calidad de investigador cultural que resuelve problemas de aula concretos concernientes a las necesidades de sus alumnos, y que los estudiantes precisan 
de tutelas, andamiajes o mediaciones, así como de mecanismos cognitivos y metacognitivos de escritura, revisión y reescritura; y por otra, que la competencia escrita no está siempre relacionada con la formación profesional y ciudadana de los universitarios. Tal vez desde esa lente multiangular y altamente demandante, los profesores en formación sabrían cuándo, cómo, dónde, sobre qué hablar; dicho en otras palabras, serían estratégicos o competentes.

Escribir en la universidad y aprender para enseñar a escribir significa, por ende, acceder al conocimiento para leer el mundo, planificar el texto en virtud de objetivos deliberados, redactar versiones intermedias y definitivas, revisar esas versiones desde modalidades diversas, reescribir una vez más, editar para perfeccionar y divulgar ante comunidades concretas. Esto, sin duda, nos permite entender el ser mismo de los maestros en formación y proceder en la búsqueda inacabada de vías para desplegar las habilidades comunicativas de los actores de aula, siempre cruciales en el hecho didáctico. Un horizonte desafiante que, en todo caso, apunta a la preparación de escritores expertos o maduros, autónomos, en lugar de heterónomos, estratégicos o competentes, capaces de aprender a aprender y de aprender a conocer en el circuito de las exigencias escriturales de la cultura académica; y que, a todas luces, focaliza su atención en el acto de educar escritores que marchen en compañía de sus docentes, pero que, progresivamente, tengan control de sus aprendizajes y vean al profesor como alguien innecesario.

\section{REFERENCIAS}

1. Agosto, S.; Álvarez, T.; Hilario, P.; Mateo, T. y Uribe, G. (Coords.) (2017). Géneros discursivos y estrategias para redactar textos académicos en Secundaria. Barcelona: Octaedro.

2. Alvarado, J., Ramírez, J. y Villarreal, J. (2016). Alfabetización académica: Herramienta conceptual para comprender la producción escrita de estudiantes de posgrado en educación. Recuperado el 23 de enero de 2020, de https://www.academia.edu/34762919/Alfabetizaci\%C3\%B3n acad\%C3\%A9mica_Herramienta_conceptual_para_comprender_la_producci\% $\% 3 \%$ B3n_escrita_ de_estudiantes_de_posgrado_en_educaci\% $3 \% \mathrm{~B} 3 \mathrm{n}$

3. Aristóteles (1986). El arte de la retórica. Buenos Aires: Eudeba.

4. Bachman, L. (1995). Habilidad lingüística comunicativa. En M. Llobera (Coord.), Competencia comunicativa: documentos básicos en la enseñanza de lenguas extranjeras (págs. 105-128). Madrid: Edelsa.

5. Boillos, M. (2017). Escribir a través de los géneros discursivos en el marco educativo basado en las competencias: el caso del País Vasco. Tejuelo, 26, págs. 63-90. https://doi.org/10.17398/19888430.26 .63

6. Canale, M. y Swain, M. (1980). Fundamentos teóricos de los enfoques comunicativos. La enseñanza y la evaluación de una segunda lengua. Signos. Teoría y práctica de la educación, 17, págs. 54-62. Recuperado de http://www.quadernsdigitals.net/datos/hemeroteca/r_3/nr_46/a_673/673.html

7. Carlino, P. (2009). Exploración de géneros, diario de tesis y revisión entre pares: Análisis de un ciclo de investigación-acción en talleres de tesis de posgrado. En E. Narvaja (Coord.), Escritura y producción de conocimiento en las carreras de posgrado (págs. 227-249). Buenos Aires: Santiago Arcos. Recuperado de https://www.aacademica.org/paula.carlino/182.pdf

8. Cicerón (1997). De la invención retórica (trad. B. Reyes). México: Universidad Nacional Autónoma de México.

9. Didactext (Grupo) (2003). Modelo sociocognitivo, pragmalingüístico y didáctico para la producción de textos escritos. Didáctica. Lengua y Literatura, 15, págs. 77-104. Recuperado de https://revistas.ucm.es/index.php/DIDA/article/view/DIDA0303110077A

10. Didactext (Grupo) (2015). Nuevo marco para la producción de textos académicos. Didáctica. Lengua y Literatura, 27, págs. 219-254. https://doi.org/10.5209/rev_DIDA.2015.v27.50871

11. Elliott, J. (2000). La investigación acción en educación. Madrid: Morata. 
12. Guerrero, H. y Chois, P. (2019). Prácticas de escritura en posgrado: el caso de una maestría en educación. Lenguaje, 47 (1), págs. 120-146. https://doi.org/10.25100/lenguaje.v47i1.6094

13. Gumpers, J. y Hymes, D. (1972). Directions in sociolinguistics. The ethnography of communication. New York: Basil Blackwell.

14. Hymes, D. (1971). On communicative competence. En J. Pride y J. Colmes (Eds.), Sociolinguistics. Selected Readings (págs. 269-293). Harmondsworth: Penguin.

15. Mercer, N. y Littleton, K. (2007). Dialogue and the development of children's thinking: Asociocultural approach. Londres: Routledge. https://doi.org/10.4324/9780203946657

16. Narváez, E. (2017). Las teorías de los géneros discursivos en el campo de la lectura y la escritura en la educación superior: análisis de datos emergentes de artículos publicados en una revista científica colombiana. Acción Pedagógica, 26 (1), págs. 70-87. Recuperado de http://www.saber. ula.ve/handle/123456789/44474.

17. Uribe, G.; Parra, E. y Zambrano-Valencia, J. (2019). Producción de ensayos y artículos de divulgación en asignaturas de lengua castellana: Implicaciones en la formación del profesorado. Lenguaje, 47 (2S), págs. 569-598. http://dx.doi.org/10.25100/lenguaje.v47i3.6980 